\title{
Rich dynamics of a food chain model with ratio-dependent type III functional responses
}

\author{
Manju Agarwal and Vimlesh Singh \\ Department of Mathematics \& Astronomy, Lucknow University, Lucknow-226007, INDIA. \\ *Corresponding Author: Emails:(manjuak@yahoo.co.in,ManjuAgarwal),and(aryansmath.lko@gmail.com,Vimlesh)
}

\begin{abstract}
This paper deals the dynamics of a tri trophic food chain model with ratio-dependent type III functional response. The investigations that are presented in this paper focus on the computation of food chain with and without time delay. Two types of discrete time delay in top level predator population are considered. In first type time delay may be regarded as a delay due to reaction time or gestation period of the top predator. In second type, delay may introduce in reaction term of top predator population and it assumes that the change rate of predator depends upon the number of prey and of the number of the predators present in some previous time. In absence of delay, the conditions for boundedness of the system are established. Stability analysis of model is carried out by using usual theory of ordinary differential equation. Further, it is proved that the system undergoes Hopf bifurcations, using delay as a bifurcating parameter. We have also shown that Hopf bifurcation may also occur when delay passes its critical value. Finally, our study shows that time delay plays a significant role on the stability of the system. It breaks the stable behaviour of model and drives it to unstable state.
\end{abstract}

Keywords: Food chain model, Ratio-dependent, Boundedness, Stability, Time-delay, Hopf bifurcation.

\section{Introduction}

The debates about ratio-dependent predation (Arditi and Ginzburg, 1989; Arditi et al., 1991; Abrams and Ginzburg 2000) have drawn ecologists' attention on the issue of correctly specifying the functional response (the instantaneous rate of prey consumption per predator). As the link between predator and prey dynamics, the functional response is necessarily critical for predator - prey interactions and it is also important for the dynamics of food chain or food web such as the response of lakes, bio control of pest (Hsu et al., 2003). The choice of particular functional form to model a process rate can have surprising effects on statistical inference and prediction. In 2002, Jost and Ellner presented a method to remove the limitations by reconstructing the functional response non - parametrically from predator-prey time series data. They use this method to data on a protozoan predator-prey interactions, and obtain significant evidence of predator dependence in functional response. The crucial element in their analysis is to include time-lags in the prey and predator reproduction rates, and show that these delays improve to fit the model significantly. Finally, compare the different non-parametrically reconstructed functional response to parametric form. They introduced and analyzed a two species model with ratio-dependent type III functional responses. In this paper, we have considered the model for three species food chain with ratio-dependent type III functional response.

A simple multispecies system comprising of three species forming a food chain was discussed by Freedman (1977). Food chains and webs in the environment are highly complex and interdependent systems. Seemingly insignificant changes in the parameter of such system can have drastic consequences. Food chain can be modeled by the system of ordinary differential equations that approximate species or functional feeding group behaviour with a variety of functional responses. Many simple two species food chain models have been thoroughly explored, while new discoveries continue to be made in examining models with three or four trophic levels (e.g. Moghadas and Gummel 2003). Hsu et al. (2003) studied a ratio-dependent food chain model with Michalies - 
Menten type functional response. They presented that food chain model is rich in boundary dynamics and capable of generating extinction dynamics and the successful implementations of biological controls. In many field situations, plant-herbivore-parasitoid food chains have become extremely important and it has been shown that parasitoids may determine fitness of the plant and destroying herbivores (Fritzche-Hoballahel et.al. 2001; Loon and Boer 2000 and Gomez et.al., 1994). Also to reduce the indiscriminate use of pesticides, recently tea scientists are using predators or pathogens to control the pests of tea. Thus three species system like plant-herbivore-parasitoid, plant-pest-predator et cetera is emerging in different branches of biology in their own right.

However in this paper we have analyzed the dynamics of tri-trophic food chain composed of a prey $X$, a middle predator $Y$ and a super predator (or top predator) $Z$. An important factor in modeling of tri trophic food chain is the choice of functional responses governing the prey-predator and predator-super predator interactions. Here we have taken a general predator prey super predator model with ratio-dependent III type functional response. Jost and Ellner (2000) introduced and analyzed a two species model with ratio-dependent III type functional responses. We have generalized that model for three species.

Actions and reactions take time to effect in real life problems. In this context a simple and natural way to understand predatorprey dynamics is to incorporate discrete delay into the predator equations. Volterra (1926) first include the delay in prey-predator model, who took in to account time taken for pollutants produced by a population to build up, eventually increasing the death rate of the population. Delay may also be due to development time of the population itself or of its resources. Kuang (1993) mentioned that animals must take time to digest their food before further activities and responses take place and hence any model of species dynamics without delays is an approximation at best. Detailed arguments on importance and usefulness of time delays in realistic models may be found in classical books of Gopalsamy (1992), Macdonald (1989) and Kuang (1993). There are many different kinds of delayed predator-prey models in the literature. Let $N_{1}(t)$ and $N_{2}(t)$ denote the prey and predator population densities at time $t$, respectively. For the generalized Gauss-type predator prey model of the following form:

$$
\begin{aligned}
\frac{d N_{1}}{d t} & =N_{1}(t) f\left(N_{1}(t)\right)-N_{2}(t) g\left(N_{1}(t)\right), \\
\frac{d N_{2}}{d t} & =N_{2}(t)\left[-d+h\left(N_{1}(t)\right)\right] .
\end{aligned}
$$

Basically, a constant time-delay can be incorporated in to the model in three different ways.

1. A time-delay $\tau$ in the prey specific growth term $f\left(N_{1}(t)\right)$, that is,

$$
\begin{aligned}
\frac{d N_{1}}{d t} & =N_{1}(t) f\left(N_{1}(t-\tau)\right)-N_{2}(t) g\left(N_{1}(t)\right), \\
\frac{d N_{2}}{d t} & =N_{2}(t)\left[-d+h\left(N_{1}(t)\right)\right] .
\end{aligned}
$$

System (b) is proposed based on the assumption that in the absence of predator the prey satisfies the Hutchinson's equation.

2. A time-delay $\tau$ in the predator response term $h\left(N_{1}(t)\right)$ in the predator equation, that is,

$$
\begin{aligned}
\frac{d N_{1}}{d t} & =N_{1}(t) f\left(N_{1}(t-\tau)\right)-N_{2}(t) g\left(N_{1}(t)\right), \\
\frac{d N_{2}}{d t} & =N_{2}(t)\left[-d+h\left(N_{1}(t-\tau)\right)\right] .
\end{aligned}
$$

The delay in system (c) can be regarded as a delay due to gestation period or reaction time of the predators. Kuang (1993), Kuang and Beretta (1996), Samanta (2000) and others has been studied the system with this type of delay.

3. A time-delay $\tau$ in the interaction term $N_{2}(t) h\left(N_{1}(t)\right)$ of the predator equation, that is,

$$
\begin{aligned}
\frac{d N_{1}}{d t} & =N_{1}(t) f\left(N_{1}(t-\tau)\right)-N_{2}(t) g\left(N_{1}(t)\right), \\
\frac{d N_{2}}{d t} & \left.=-d N_{2}(t)+N_{2}(t-\tau) h\left(N_{1}(t-\tau)\right)\right] .
\end{aligned}
$$

System (d) assumes the change rate of the predator depends on the number of prey and of the predators present at some previous time. The well known Wangersky and Cumingham model (1957) is such a model.

The rest of the paper is organized as follows. In section 2, we present a brief sketch of the construction of the model. In section 3 , we have determined the boundary equilibrium point and their stabilities; the boundedness of our model is also studied in this section. In section 4, we have determined the necessary and sufficient condition for the existence of interior equilibrium point and study its stability. It is seen that the top predator-free boundary equilibrium point possesses nonempty stable and unstable manifold whenever the interior equilibrium point exists. The effect of discrete time-delay on the system is investigated in section (5). Computer simulations of variety of numerical solutions of system with delay are presented and a study of the occurrence of Hopf 
bifurcation is presented in section (6). At last general discussions of the paper and biological implications of our model are presented in section 7 .

\section{Mathematical Model}

Model that we analyze in this paper, describe a tri trophic food chain. This food chain is composed of a prey, whose population density is denoted by $X$, a predator whose population density is denoted by $Y$ and a super predator (or top predator) whose population density is denoted by $Z$. Before introducing the model, we would like to present a brief sketch of the construction of model. This may indicate the biological relevance of it. Behaviour of the entire community is assumed to arise from the coupling of these interacting species. Among these three species have simple relation $Z$ prey on $Y$ and only $Y$ and $Y$ prey on $X$ and nutrient recycling is not accounted for. This simple relation produces the so-called simple food chain. This is an interesting practical assumption from both mathematical and biological point of view. For example, in a waste treatment process, the bacteria lives on the waste (or nutrient) while other organism as ciliates feed on the bacteria (Kuang (2000)). Also in the tea plant -pest predator interaction, the pest specializes on the tea plant and predators destroy pests by feeding on them (Das et.al. 1998, Das and Barua 1990 and Kabir 2001).

It is mentioned that an important factor in modeling of tritrophic food chain is the choice of functional response governing the prey-predator - super-predator interactions. Here we have considered a ratio-dependent type III functional response for both prey - predator and predator-top predators. Therefore, mathematical models governing the system of nonlinear ordinary differential equations are

$$
\begin{array}{lrl}
\frac{d X}{d T}=r X\left(1-\frac{X}{k}\right)-\frac{1}{\eta_{1}} \frac{m_{1} X^{2} Y}{a_{1} Y^{2}+X^{2}}, & X(0)>0, \\
\frac{d Y}{d T}=\frac{m_{1} X^{2} Y}{a_{1} Y^{2}+X^{2}}-d_{1} Y-\frac{1}{\eta_{2}} \frac{m_{2} Y^{2} Z}{a_{2} Z^{2}+Y^{2}}, & Y(0)>0, \\
\frac{d Z}{d T}=\frac{m_{2} Y^{2} Z}{a_{2} Z^{2}+Y^{2}}-d_{2} Z, & Z(0)>0,
\end{array}
$$

Here for $i=1,2, \eta_{i}, a_{i}, d_{i}$ and $m_{i}$ are yield constants, half saturation constants, predators natural death rates and maximal predator growth rates respectively. $r$ and $k$ are the prey intrinsic growth rate and carrying capacity respectively. We make an obvious assumption that all the parameters are positive. Since the densities of the population cannot be negative, the state space of system (1) is given by

$$
R_{+}^{3}=\left\{(X, Y, Z) \in R^{3}, X \geq 0, Y \geq 0, Z \geq 0\right\} .
$$

Model (1) has 10 parameters in all, which make mathematical analysis complex. So, to reduce number of parameters, model is non-dimensionalized by using

$$
t \rightarrow r \mathrm{~T}, \quad x \rightarrow \frac{X}{k}, \quad y \rightarrow \frac{\sqrt{a_{1}} Y}{k}, \quad z \rightarrow \frac{\sqrt{a_{1} a_{2}} Z}{k}
$$

Then the system (1) takes the form

$$
\begin{array}{ll}
x^{\prime}(t)=x(1-x)-\frac{c_{1} x^{2} y}{x^{2}+y^{2}}, & x(0)>0, \\
y^{\prime}(t)=\frac{p_{1} x^{2} y}{x^{2}+y^{2}}-q_{1} y-\frac{c_{2} y^{2} z}{y^{2}+z^{2}}, & y(0)>0, \\
z^{\prime}(t)=-q_{2} z+\frac{p_{2} y^{2} z}{y^{2}+z^{2}}, & z(0)>0,
\end{array}
$$

Where $\left('=\frac{d}{d t}\right), \quad c_{1}=\frac{m_{1}}{\eta_{1} r \sqrt{a_{1}}}, \quad p_{1}=\frac{m_{1}}{r}, \quad q_{1}=\frac{d_{1}}{r}, \quad p_{2}=\frac{m_{2}}{r}, \quad q_{2}=\frac{d_{2}}{r}, \quad c_{2}=\frac{m_{2}}{\eta_{2} \sqrt{a_{2} r}}$

\section{Boundedness of Solutions}

Theorem (3.1):- The solutions $x(t), y(t)$ and $z(t)$ of system (2) initiating in $R_{+}^{3}$ are positive and bounded for all $t \geq 0$. 
Proof:- Since the densities of the population can never be negative, therefore obviously the solutions $x(t), y(t)$ and $z(t)$ are positive for all $t \geq 0$.

Since $x^{\prime}(t) \leq x(1-x)$,

So we have $\lim _{t \rightarrow \infty} \sup x(t) \leq 1$.

Consider, $\quad L=\frac{p_{1}}{c_{1}} x+y+\frac{c_{2}}{p_{2}} z$

Then,

$$
\begin{aligned}
& \frac{d L}{d t}=\frac{p_{1}}{c_{1}} x(1-x)-q_{1} y-\frac{c_{2}}{p_{2}} q_{2} z \\
& \leq \frac{p_{1}}{c_{1}} x-q_{1} y-\frac{c_{2}}{p_{2}} q_{2} z, \\
& \leq \frac{2 p_{1}}{c_{1}}-\delta L, \quad \text { where, } \delta=\min \left\{1, q_{1}, q_{2}\right\}
\end{aligned}
$$

Therefore,

$$
\frac{d L}{d t}+\delta L \leq \frac{2 p_{1}}{c_{1}} .
$$

Applying a Lemma on differential inequalities \{Birkhoff and Rota (1982)\}, we obtain

$$
0 \leq L(x, y, z) \leq \frac{2 p_{1}}{c_{1} \delta}+\frac{L(x(0), y(0), z(0))}{e^{\delta t}},
$$

and for $t \rightarrow \infty$,

$$
0 \leq L \leq \frac{2 p_{1}}{c_{1} \delta} .
$$

Thus, all solutions of system (2) enter into the region:

$$
B=\left\{(x, y, z): 0 \leq x \leq 1,0 \leq L \leq \frac{2 p_{1}}{c_{1} \delta}+\varepsilon \text {, for any } \varepsilon>0\right\}
$$

Hence theorem is proved.

\subsection{Boundary Equilibria and Stability}

In this section, we study the existence and local stability analysis of boundary equilibrium point $\hat{E}(\hat{x}, \hat{y}, 0)$ of system (2). The boundary equilibrium point $\hat{E}$ in $x-y$ plane is positive solution of following algebraic equations

$$
\begin{gathered}
1-x-\frac{c_{1} x y}{x^{2}+y^{2}}=0, \\
\frac{p_{1} x^{2}}{x^{2}+y^{2}}-q_{1}=0,
\end{gathered}
$$

Solving above equations we get

$$
\begin{aligned}
& \hat{y}=\left(\frac{p_{1}-q_{1}}{q_{1}}\right)^{1 / 2} \hat{x}, \\
& \hat{x}=\frac{p_{1}-c_{1}\left(p_{1}-q_{1}\right)^{1 / 2} q_{1}^{1 / 2}}{p_{1}} .
\end{aligned}
$$

Exist if $p_{1}>q_{1}$ and

$$
p_{1}>c_{1}\left(p_{1}-q_{1}\right)^{1 / 2} q_{1}^{1 / 2}
$$


On the question of stability of $\hat{E}(\hat{x}, \hat{y}, 0)$, we have determined the variational matrix $M(\hat{E})$ at $\hat{E}(\hat{x}, \hat{y}, 0)$ as

$$
M(\hat{E})=\left[\begin{array}{ccc}
\hat{x}\left(-1-\frac{c_{1} \hat{y}\left(\hat{y}^{2}-\hat{x}^{2}\right)}{\left(\hat{x}^{2}+\hat{y}^{2}\right)^{2}}\right) & -\frac{c_{1} \hat{x}^{2}\left(\hat{x}^{2}-\hat{y}^{2}\right)}{\left(\hat{x}^{2}+\hat{y}^{2}\right)^{2}} & 0 \\
\frac{2 p_{1} \hat{x} \hat{y}^{3}}{\left(\hat{x}^{2}+\hat{y}^{2}\right)^{2}} & \frac{-2 p_{1} \hat{x}^{2} \hat{y}^{2}}{\left(\hat{x}^{2}+\hat{y}^{2}\right)^{2}} & -c_{2} \\
0 & 0 & p_{2}-q_{2}
\end{array}\right],
$$

Corresponding characteristic equation for equilibrium point $\hat{E}$ is $\left(\hat{\lambda}^{2}-D_{1} \hat{\lambda}+D_{2}\right)\left(\hat{\lambda}-p_{2}-q_{2}\right)=0$

Where

$$
\begin{aligned}
& D_{1}=-\hat{x}-\left(\frac{p_{1}-q_{1}}{q_{1}}\right)^{1 / 2}\left[\frac{2 p_{1}\left(p_{1}-q_{1}\right)^{1 / 2} q_{1}^{3 / 2}-c_{1} q_{1}^{2}\left(2-\frac{p_{1}}{q_{1}}\right)}{\left(\hat{x}^{2}+\hat{y}^{2}\right)^{2}}\right]<0 \\
& D_{2}=\frac{2 p_{1} \hat{x}^{3} \hat{y}^{2}}{\left(\hat{x}^{2}+\hat{y}^{2}\right)^{2}}>0
\end{aligned}
$$

Clearly, eigenvalue $\hat{\lambda}=p_{2}-q_{2}$ is positive corresponding to $z$ - direction, and other two eigenvalues are negative corresponding to $x-y$ plane. Therefore equilibrium $\hat{E}$ is stable in $x-y$ plane but unstable in $z$ direction.

\section{Interior Equilibrium Point: Its Existence and Stability}

It is obvious that the interior equilibrium point $E^{*}\left(x^{*}, y^{*}, z^{*}\right)$ of system (2) exists in the interior of the first octant if there is a positive solution of following algebraic equations

$$
\begin{aligned}
& 1-x^{*}-\frac{c_{1} x^{*} y^{*}}{x^{2^{*}}+y^{2^{*}}}=0, \\
& \frac{p_{1} x^{2^{*}}}{x^{2^{*}}+y^{2^{*}}}-q_{1}-\frac{c_{2} y^{*} z^{*}}{y^{2^{*}}+z^{2^{*}}}=0, \\
& \frac{p_{2} y^{2^{*}}}{y^{2^{*}}+z^{2^{*}}}-q_{2}=0 .
\end{aligned}
$$

Thus, by solving above equations, we get

$$
\begin{aligned}
& x^{*}=1-\frac{c_{1}\left(p_{1}-B\right)^{1 / 2} B^{1 / 2}}{p_{1}} \\
& y^{*}=\left(\frac{p_{1}-B}{B}\right)^{1 / 2} x^{*} \\
& z^{*}=\left(\frac{p_{2}-q_{2}}{q_{2}}\right)^{1 / 2} y^{*}
\end{aligned}
$$


Where, $B=q_{1}+\frac{c_{2}}{p_{2}}\left(p_{2}-q_{2}\right)^{1 / 2} q_{2}^{1 / 2}$

It can be seen that $E^{*}\left(x^{*}, y^{*}, z^{*}\right)$ exists if following conditions are satisfied

(i) $p_{2}>q_{2}$

(ii) $p_{1}>B$

(iii) $p_{1}>c_{1}\left(p_{1}-B\right)^{1 / 2} B^{1 / 2}$

\subsection{Local Stability Analysis}

Now to investigate the local stability of interior equilibrium $E^{*}\left(x^{*}, y^{*}, z^{*}\right)$, we first find the variational matrix $M\left(E^{*}\right)$ at interior equilibrium point

$$
M\left(E^{*}\right)=\left[\begin{array}{ccc}
b_{11} & b_{12} & 0 \\
b_{21} & b_{22} & b_{23} \\
0 & b_{32} & b_{33}
\end{array}\right],
$$

Where $b_{11}=x^{*}\left(-1+\frac{c_{1} y^{*}\left(x^{* 2}-y^{* 2}\right)}{\left(x^{* 2}+y^{* 2}\right)^{2}}\right), \quad b_{12}=-\frac{c_{1} x^{* 2}\left(x^{* 2}-y^{* 2}\right)}{\left(x^{* 2}+y^{* 2}\right)^{2}}, \quad b_{21}=\frac{2 p_{1} x^{*} y^{* 3}}{\left(x^{*^{2}}+y^{*^{2}}\right)^{2}}$,

$b_{22}=\frac{-2 p_{1} x^{*^{2}} y^{*^{2}}}{\left(x^{*^{2}}+y^{*^{2}}\right)^{2}}+\frac{c_{2} y^{*} z^{*}\left(y^{*^{2}}-z^{*^{2}}\right)}{\left(y^{*^{2}}+z^{*^{2}}\right)^{2}}, b_{23}=-\frac{c_{2} y^{*^{2}}\left(y^{*^{2}}-z^{* 2}\right)}{\left(y^{*^{2}}+z^{*^{2}}\right)^{2}}, b_{32}=\frac{2 p_{2} y^{*} z^{* 3}}{\left(y^{*^{2}}+z^{*^{2}}\right)^{2}}, b_{33}=-\frac{2 p_{2} y^{*^{2}} z^{*^{2}}}{\left(y^{*^{2}}+z^{*^{2}}\right)^{2}}$.

Corresponding characteristic equation becomes

$$
\lambda^{3}+A_{1} \lambda^{2}+A_{2} \lambda+A_{3}=0
$$

Where

$$
\begin{aligned}
& A_{1}=-\left(b_{11}+b_{22}+b_{33}\right), \\
& A_{1}=x^{*}+\frac{c_{1} x^{*} y^{*}\left(y^{* 2}-x^{* 2}\right)}{\left(x^{* 2}+y^{* 2}\right)^{2}}+\frac{2 p_{1} x^{* 2} y^{* 2}}{\left(x^{* 2}+y^{* 2}\right)^{2}}+\frac{c_{2} y^{*} z^{*}\left(z^{* 2}-y^{* 2}\right)}{\left(y^{* 2}+z^{* 2}\right)^{2}}+\frac{2 p_{2} y^{* 2} z^{* 2}}{\left(y^{* 2}+z^{* 2}\right)^{2}} \\
& A_{2}=b_{22} b_{33}+b_{11} b_{22}+b_{11} b_{33}-b_{12} b_{21}-b_{23} b_{32}, \\
& A_{2}=\frac{4 p_{1} p_{2} x^{* 2} y^{* 4} z^{* 2}+c_{1} c_{2} x^{*} y^{* 2} z^{*}\left(y^{* 2}-z^{* 2}\right)\left(x^{* 2}-y^{* 2}\right)}{\left(x^{* 2}+y^{* 2}\right)^{2}\left(y^{* 2}+z^{* 2}\right)^{2}} \frac{2 p_{1} x^{* 3} y^{* 2}}{\left(x^{* 2}+y^{* 2}\right)^{2}} \\
& +\frac{2 p_{2} x^{*} y^{* 2} z^{* 2}-c_{2} x^{*} y^{*} z^{*}\left(y^{* 2}-z^{* 2}\right)}{\left(y^{* 2}+z^{* 2}\right)^{2}}-\frac{2 p_{2} c_{1} x^{*} y^{* 3} z^{* 2}\left(x^{* 2}-y^{* 2}\right)}{\left(x^{* 2}+y^{* 2}\right)^{2}\left(y^{* 2}+z^{* 2}\right)^{2}} \\
& A_{3}=\operatorname{det} M\left(E^{*}\right) \\
& =\frac{4 p_{1} p_{2} x^{* 3} y^{* 4} z^{* 2}}{\left(x^{* 2}+y^{* 2}\right)^{2}\left(y^{* 2}+z^{* 2}\right)^{2}}>0,
\end{aligned}
$$

Now

$$
\begin{aligned}
& \Delta=A_{1} A_{2}-A_{3}=-\left(b_{11}\right)^{2} b_{22}-\left(b_{11}\right)^{2} b_{33}+b_{11} b_{12} b_{21}-b_{22}\left(b_{33}\right)^{2}-b_{11}\left(b_{22}\right)^{2}-2 b_{11} b_{22} b_{33}+b_{22} b_{12} b_{21} \\
& +b_{23} b_{32} b_{22}-\left(b_{22}\right)^{2} b_{33}-b_{11}\left(b_{33}\right)^{2}+b_{23} b_{32} b_{33}
\end{aligned}
$$

Therefore, an application of Routh-Hurwitz criterion shows that 
If $\quad b_{11}<0$ and $b_{22}<0$

Then the following conditions are satisfied:

$$
A_{1}>0, A_{3}>0 \text { and } A_{1} A_{2}-A_{3}>0 \text {. }
$$

Hence, positive equilibrium point $E^{*}$ is locally asymptotically stable under the condition (15a) and (15b).

\section{Model with discrete delay}

It is already mentioned that time delay is an important factor in biological as well as ecological systems. Examine the experimental findings on the interaction of larval Thanasimus dubius (cleried beetle) and Dendroctonus fronttalis (bark beetle) during attack of host tree. Reeve ,1997; suggested that a model including ratio - dependence and time delay for $T$. dubies and $D$. frontalis interaction that determine the net stabilizing and destabilizing effect on $D$. frontalis dynamics. Therefore as a starting point, we consider the model involving two types of discrete time delay. In the first model there is delay in the predator response function, while in the second one is the well known Wangersky Cunningham type model, 1957; this assumes that the change in the number of top predator depends upon the number of middle predator and top predator present in some previous time.

\section{Type I Delay}

$$
\begin{aligned}
& x^{\prime}(t)=x(1-x)-\frac{c_{1} x^{2} y}{x^{2}+y^{2}}, \\
& y^{\prime}(t)=\frac{p_{1} x^{2} y}{x^{2}+y^{2}}-q_{1} y-\frac{c_{2} y^{2} z}{y^{2}+z^{2}}, \\
& z^{\prime}(t)=z\left[-q_{2}+\frac{p_{2} y^{2}(t-\tau)}{y^{2}(t-\tau)+z^{2}(t-\tau)}\right] .
\end{aligned}
$$

The delay $\tau \neq 0$ in (16) can be regarded as a delay due to reaction time of the top predator $z$. System (16) has same equilibria as system (2) has. The main purpose of this section to study the stability behaviour of $E^{*}\left(x^{*}, y^{*}, z^{*}\right)$ in the presence of discrete delay $(\tau \neq 0)$. Now to prove the stability behaviour of $E^{*}\left(x^{*}, y^{*}, z^{*}\right)$ for the system (16), first we lineraize the system (16) by using following transformation

$$
\begin{aligned}
& x(t)=x^{*}+u(t), \\
& y(t)=y^{*}+v(t) \\
& z(t)=z^{*}+w(t) .
\end{aligned}
$$

Then linear system is given by

$\dot{u}(t)=a_{11} u(t)+a_{12} v(t)$,

$\dot{v}(t)=a_{21} u(t)+a_{22} v(t)+a_{23} w(t)$,

$\dot{w}(t)=b_{32} v(t-\tau)+b_{33} w(t-\tau)$.

Where, $a_{11}=x^{*}\left(-1+\frac{c_{1} y^{*}\left(x^{* 2}-y^{* 2}\right)}{\left(x^{* 2}+y^{* 2}\right)^{2}}\right), a_{12}=-\frac{c_{1} x^{* 2}\left(x^{* 2}-y^{* 2}\right)}{\left(x^{* 2}+y^{* 2}\right)^{2}}, a_{21}=\frac{2 p_{1} x^{*} y^{* 3}}{\left(x^{* 2}+y^{* 2}\right)^{2}}$

$a_{22}=\frac{-2 p_{1} x^{* 2} y^{* 2}}{\left(x^{* 2}+y^{* 2}\right)^{2}}+\frac{c_{2} y^{*} z^{*}\left(y^{* 2}-z^{* 2}\right)}{\left(y^{* 2}+z^{* 2}\right)^{2}}, a_{23}=-\frac{c_{2} y^{*^{2}}\left(y^{*^{2}}-z^{* 2}\right)}{\left(y^{* 2}+z^{* 2}\right)^{2}}, b_{32}=\frac{2 p_{2} y^{*} z^{* 3}}{\left(y^{* 2}+z^{* 2}\right)^{2}}, b_{33}=-\frac{2 p_{2} y^{*^{2}} z^{*^{2}}}{\left(y^{* 2}+z^{*^{2}}\right)^{2}}$.

We look for solution of the model (16) of the form $A(t)=\rho e^{-\lambda t}, \rho \neq 0$, this leads to the characteristic equation

$\Delta(\lambda, \tau)=\lambda^{3}+P_{1} \lambda^{2}+P_{2} \lambda+\left(P_{3} \lambda^{2}+P_{4} \lambda+P_{5}\right) e^{-\lambda \tau}=0$

Where, $P_{1}=-\left(a_{11}+a_{22}\right)$,

$P_{2}=a_{11} a_{22}-a_{12} a_{21}$,

$P_{3}=-b_{33}$, 
$P_{4}=\left(a_{11}+a_{22}\right) b_{33}-a_{23} b_{32}$,

$P_{5}=a_{12} a_{21} b_{33}-a_{11} a_{22} b_{33}+a_{11} a_{23} b_{32}$.

The eigenvalues are the roots of the characteristic equation (17) of the system (16), that has infinitely many solutions. We wish to find periodic solution of the system, for the periodic solution eigenvalues will be purely imaginary so we substitute $\lambda=i \omega, \omega>0$ in equation (17), we get $-i \omega^{3}-P_{1} \omega^{2}+i \omega P_{2}+\left(-P_{3} \omega^{2}+i \omega P_{4}+P_{5}\right) e^{-i \omega \tau}=0$

Comparing real and imaginary parts, we get

$P_{1} \omega^{2}=\left(P_{5}-P_{3} \omega^{2}\right) \cos \omega \tau+\omega P_{4} \sin \omega \tau$

$-\omega^{3}+P_{2} \omega=-\omega P_{4} \cos \omega \tau+\left(P_{5}-P_{3} \omega^{2}\right) \sin \omega \tau$

Squaring and adding (18a) and (18b), we get

$\omega^{6}+d_{1} \omega^{4}+d_{2} \omega^{2}+d_{3}=0$

Where $d_{1}=P_{1}^{2}-2 P_{2}-P_{3}^{2}, d_{2}=P_{2}{ }^{2}+2 P_{3} P_{5}-P_{4}^{2}, d_{3}=-P_{5}^{2}$.

Substituting $\omega^{2}=\delta$, equation (18c) becomes

$F(\delta)=\delta^{3}+d_{1} \delta^{2}+d_{2} \delta+d_{3}=0$

By Descarte's rule of sign, the cubic equation (18d) has at least one positive root. Consequently the stability criteria of the system for $\tau=0$ will not necessarily ensure the stability of system for $\tau \neq 0$.

Again solving (18a) and (18b), we get a critical value of delay that is given as follows

$\cos \omega \tau=\frac{\omega^{4}\left(P_{4}-P_{1} P_{3}\right)+\omega^{2}\left(P_{5} P_{1}-P_{2} P_{4}\right)}{\omega^{2} P_{4}^{2}+\left(P_{5}-P_{3} \omega^{2}\right)^{2}}$,

So, corresponding to $\lambda=i \omega_{0}$, there exist $\tau_{0 n}$ such that

$\tau_{0 n}=\frac{1}{\omega_{0}}\left[\cos ^{-1}\left(\frac{\omega_{0}^{4}\left(P_{4}-P_{1} P_{3}\right)+\omega_{0}{ }^{2}\left(P_{5} P_{1}-P_{2} P_{4}\right)}{\omega_{0}{ }^{2} P_{4}{ }^{2}+\left(P_{5}-P_{3} \omega_{0}{ }^{2}\right)^{2}}\right)\right]+\frac{2 n \pi}{\omega_{0}}, \quad n=0,1,2,3 \ldots$

\section{Hopf Bifurcation}

We observe that the conditions for Hopf bifurcation (Hale and Lunel, (1993)) are satisfied yielding the required periodic solution, that is, $\left[\frac{d(\operatorname{Re} \lambda)}{d \tau}\right]_{\tau=\tau_{0}} \neq 0$

This signifies that there exists at least one eigenvalue with positive real part for $\tau>\tau_{0}$.

Now we show the existence of Hopf bifurcation near $E^{*}$, by taking $\tau$ as a bifurcating parameter.

Differentiating equation (17) with respect to $\tau$, we obtain

$$
\begin{aligned}
\left(\frac{d \lambda}{d \tau}\right)^{-1} & =\frac{3 \lambda^{2}+2 P_{1} \lambda+P_{2}}{\lambda\left(P_{3} \lambda^{2}+P_{4} \lambda+P_{5}\right) e^{-\lambda \tau}}+\frac{2 P_{3} \lambda+P_{4}}{\lambda\left(P_{3} \lambda^{2}+P_{4} \lambda+P_{5}\right)}-\frac{\tau}{\lambda} \\
& =\frac{3 \lambda^{3}+2 P_{1} \lambda^{2}+P_{2} \lambda}{\lambda^{2}\left(P_{3} \lambda^{2}+P_{4} \lambda+P_{5}\right) e^{-\lambda \tau}}+\frac{2 P_{3} \lambda^{2}+P_{4} \lambda}{\lambda^{2}\left(P_{3} \lambda^{2}+P_{4} \lambda+P_{5}\right)}-\frac{\tau}{\lambda} \\
& =\frac{2 \lambda^{3}+P_{1} \lambda^{2}-\left(P_{3} \lambda^{2}+P_{4} \lambda+P_{5}\right) e^{-\lambda \tau}}{\lambda^{2}\left(P_{3} \lambda^{2}+P_{4} \lambda+P_{5}\right) e^{-\lambda \tau}}+\frac{2 P_{3} \lambda^{2}+P_{4} \lambda}{\lambda^{2}\left(P_{3} \lambda^{2}+P_{4} \lambda+P_{5}\right)}-\frac{\tau}{\lambda} \\
& =-\frac{2 \lambda^{3}+P_{1} \lambda^{2}}{\lambda^{2}\left(\lambda^{3}+P_{1} \lambda^{2}+P_{2} \lambda\right)}+\frac{2 P_{3} \lambda^{2}+P_{4} \lambda}{\lambda^{2}\left(P_{3} \lambda^{2}+P_{4} \lambda+P_{5}\right)}-\frac{1}{\lambda^{2}}-\frac{\tau}{\lambda}
\end{aligned}
$$




$$
=-\frac{2 \lambda+P_{1}}{\left(\lambda^{3}+P_{1} \lambda^{2}+P_{2} \lambda\right)}+\frac{P_{3} \lambda^{2}-P_{5}}{\lambda^{2}\left(P_{3} \lambda^{2}+P_{4} \lambda+P_{5}\right)}-\frac{\tau}{\lambda}
$$

Taking $\lambda=i \omega_{0}$ in above equation, we get

$$
\begin{aligned}
\left(\frac{d \lambda}{d \tau}\right)_{\lambda=i \omega_{0}}^{-1} & =\frac{2 \omega_{0} i+P_{1}}{P_{1} \omega_{0}^{2}-i\left(P_{2} \omega_{0}-\omega_{0}^{3}\right)}+\frac{P_{3} \omega_{0}^{2}+P_{5}}{\omega_{0}^{2}\left(\left(P_{5}-P_{3} \omega_{0}^{2}\right)+i P_{5} \omega_{0}\right)}+\frac{\tau}{\omega_{0}} i \\
& =\frac{\left(2 \omega_{0} i+P_{1}\right)\left(P_{1} \omega_{0}^{2}+i\left(P_{2} \omega_{0}-\omega_{0}^{3}\right)\right)}{P_{1}^{2} \omega_{0}^{4}+\left(P_{2} \omega_{0}-\omega_{0}^{3}\right)^{2}}+\frac{\left(P_{3} \omega_{0}^{2}+P_{5}\right)\left(\left(P_{5}-P_{3} \omega_{0}^{2}\right)-i P_{4} \omega_{0}\right)}{\omega_{0}^{2}\left\{\left(P_{5}-P_{3} \omega_{0}^{2}\right)^{2}+P_{4}{ }^{2} \omega_{0}^{2}\right\}}+\frac{\tau}{\omega_{0}} i \\
\operatorname{Re}\left(\frac{d \lambda}{d \tau}\right)_{\lambda=i \omega_{0}}^{-1} & =\frac{\left(P_{1}^{2} \omega_{0}^{2}-2 \omega_{0}\left(P_{2} \omega_{0}-\omega_{0}^{3}\right)\right)}{P_{2}{ }^{2} \omega_{0}^{4}+\left(P_{2} \omega_{0}-\omega_{0}^{3}\right)^{2}}+\frac{P_{5}{ }^{2}-P_{3}{ }^{2} \omega_{0}^{4}}{\omega_{0}^{2}\left\{\left(P_{5}-P_{3} \omega_{0}^{2}\right)^{2}+P_{4}{ }^{2} \omega_{0}^{2}\right\}}>0
\end{aligned}
$$

Thus we obtain

$\operatorname{Re}\left(\frac{d \lambda}{d \tau}\right)_{\lambda=i \omega_{0}}^{-1}>0$

Therefore the transversality condition holds and hence Hopf bifurcation occurs at $\tau=\tau_{0}$.

Theorem (5.1): If $d_{3}<0$ and $E^{*}$ is asymptotically stable for $\tau=0$, it is impossible that it remain stable for $\tau>0$. Hence there exist $\tau_{0}>0$, such that $\tau_{0}>\tau, E^{*}$ is asymptotically stable and for $\tau_{0}<\tau, E^{*}$ is unstable and $\tau$ increases together with $\tau_{0}$, $E^{*}$ bifurcates into small amplitude periodic solution of Hopf type the value of $\tau_{0}$ is given by following equation

$\tau_{0 n}=\frac{1}{\omega_{0}}\left[\cos ^{-1}\left(\frac{\omega_{0}^{4}\left(P_{4}-P_{1} P_{3}\right)+\omega_{0}{ }^{2}\left(P_{5} P_{1}-P_{2} P_{4}\right)}{\omega_{0}{ }^{2} P_{4}{ }^{2}+\left(P_{5}-P_{3} \omega_{0}{ }^{2}\right)^{2}}\right)\right]+\frac{2 n \pi}{\omega_{0}}, \quad n=0,1,2,3 \ldots$

\section{Type II Delay}

$$
\begin{aligned}
& x^{\prime}(t)=x(1-x)-\frac{c_{1} x^{2} y}{x^{2}+y^{2}}, \\
& y^{\prime}(t)=\frac{p_{1} x^{2} y}{x^{2}+y^{2}}-q_{1} y-\frac{c_{2} y^{2} z}{y^{2}+z^{2}}, \\
& z^{\prime}(t)=\frac{p_{2} y^{2}(t-\tau) z(t-\tau)}{y^{2}(t-\tau)+z^{2}(t-\tau)}-q_{2} z .
\end{aligned}
$$

Here, the time delay in the interaction term of the top predator equation assumes that the change rate of the predator depends upon the number of prey and of the predators present at some previous time. By similar process of the model (18), we show the stability behaviour of $E^{*}\left(x^{*}, y^{*}, z^{*}\right)$ for the system (21) by using following transformations

$$
x(t)=x^{*}+u_{1}(t), \quad y(t)=y^{*}+v_{1}(t), \quad z(t)=z^{*}+w_{1}(t) .
$$

We determine the variational matrix of the system (21) at $E^{*}$ to explore local stability of mathematical model (21) with delay

$$
M=\left[\begin{array}{ccc}
c_{11} & c_{12} & 0 \\
c_{21} & c_{22} & c_{23} \\
0 & d_{32} e^{-\lambda \tau} & d_{33} e^{-\lambda \tau}-c_{33}
\end{array}\right] .
$$




$$
\begin{aligned}
& c_{11}=-x^{*}+\frac{c_{1} x^{*} y^{*}\left(x^{* 2}-y^{* 2}\right)}{\left(x^{* 2}+y^{* 2}\right)^{2}}, \quad c_{12}=-\frac{c_{1} x^{* 2}\left(x^{* 2}-y^{* 2}\right)}{\left(x^{* 2}+y^{* 2}\right)^{2}}, \\
& c_{21}=\frac{2 p_{1} x^{*} y^{* 3}}{\left(x^{* 2}+y^{* 2}\right)^{2}} \\
& c_{22}=-\frac{2 p_{1} x^{* 2} y^{* 2}}{\left(x^{* 2}+y^{* 2}\right)^{2}}+\frac{c_{2} y^{*} z^{*}\left(y^{* 2}-z^{* 2}\right)}{\left(y^{* 2}+z^{* 2}\right)^{2}}, \quad c_{23}=-\frac{c_{2} y^{* 2}\left(y^{* 2}-z^{* 2}\right)}{\left(y^{* 2}+z^{* 2}\right)^{2}}, \quad c_{33}=-q_{2} \text {, } \\
& d_{32}=\frac{2 p_{2} y^{*} z^{* 3}}{\left(y^{* 2}+z^{* 2}\right)^{2}} \\
& d_{33}=\frac{p_{2} y^{*^{2}}\left(y^{*^{2}}-z^{*^{2}}\right)}{\left(y^{*^{2}}+z^{* 2}\right)^{2}} \text {. }
\end{aligned}
$$

This leads to the characteristic equation

$$
\Delta_{1}(\lambda, \tau)=\lambda^{3}+Q_{1} \lambda^{2}+Q_{2} \lambda+\left(Q_{3} \lambda^{2}+Q_{4} \lambda+Q_{5}\right) e^{-\lambda \tau}+Q_{6}=0 .
$$

Where $Q_{1}=-c_{11}-c_{22}-c_{33}$,

$$
Q_{2}=c_{11} c_{33}+c_{11} c_{22}-c_{12} c_{21}+c_{33} c_{22}, \quad Q_{3}=-d_{33}, \quad Q_{4}=\left(c_{11}+c_{22}\right) d_{33}-c_{23} d_{32} \text {, }
$$

$Q_{5}=c_{12} c_{21} d_{33}-c_{11} c_{22} d_{33}+c_{11} c_{23} d_{32}, \quad Q_{6}=c_{12} c_{21} c_{33}-c_{11} c_{22} c_{33}$.

The eigenvalues are the roots of the characteristic equation (22) of the system (21) that has infinitely many solutions. We wish to find periodic solution of the system (21), for the periodic solution, eigenvalues will be purely imaginary. Substituting $\lambda=i \eta, \eta>0$ in equation (22), we get the transcendental equation on separating real and imaginary parts of the resulting equation and then by taking $\eta^{2}=\theta$, we get a cubic equation given by

$$
\theta^{3}+S_{1} \theta^{2}+S_{2} \theta+S_{3}=0
$$

Where $S_{1}=Q_{1}{ }^{2}-2 Q_{2}-Q_{3}^{2}, S_{2}=Q_{2}^{2}+2 Q_{3} Q_{5}-2 Q_{1} Q_{6}-Q_{4}^{2}, S_{3}=Q_{6}^{2}-Q_{5}^{2}$.

Now equation (23) will have a positive root if

$S_{1}>0$ and $S_{3}<0$.

Since, the existence condition for interior equilibrium point $E^{*}\left(x^{*}, y^{*}, z^{*}\right)$ holds true, we have the condition for $S_{1}$ to be positive and $S_{3}$ to be negative. Thus, we can say that there is a unique positive root $\eta_{0}$ satisfying (24), that is, the characteristic equation (22) has a pair of purely imaginary roots of the form $\pm i \eta_{0}$. So, corresponding to $\lambda=i \eta_{0}$, there exist $\tau_{1 k}$ such that

$$
\tau_{1 k}=\frac{1}{\eta_{0}}\left[\cos ^{-1}\left(\frac{\eta_{0}{ }^{2}\left(Q_{4} \eta_{0}{ }^{2}-Q_{2} Q_{4}\right)+\left(Q_{1} \eta_{0}{ }^{2}-Q_{6}\right)\left(Q_{5}-Q_{3} \eta_{0}{ }^{2}\right)}{\eta_{0}{ }^{2} Q_{4}{ }^{2}+\left(Q_{5}-Q_{3} \eta_{0}{ }^{2}\right)^{2}}\right)\right]+\frac{2 k \pi}{\eta_{0}}, k=0,1,2,3 \ldots
$$

For $\tau=0, E^{*}\left(x^{*}, y^{*}, z^{*}\right)$ is stable if conditions (15) holds. Then by Butler's lemma [Freedman et. al.(1983)] $E^{*}\left(x^{*}, y^{*}, z^{*}\right)$ remains stable for $\tau<\tau_{1}$ and unstable for $\tau>\tau_{1}$. We also observe that the conditions for bifurcations (Hale and Lunel, (1993)) are satisfied if condition (22) holds, that is

$$
\left[\frac{d(\operatorname{Re} \lambda)}{d \tau}\right]_{\tau=\tau_{0}}>0
$$

This signifies that there exists at least one eigenvalue with positive real part for $\tau>\tau_{1}$.

Theorem (5.2): If $S_{1}>0$ and $S_{3}<0$, then $E^{*}$ is asymptotically stable for $\tau=0$, it is impossible that it remain stable for $\tau>0$. Hence there exist $\tau_{1}>0$, such that $\tau_{1}>\tau, E^{*}$ is asymptotically stable and for $\tau_{1}<\tau, E^{*}$ is unstable and $\tau$ increases together with $\tau_{1}, E^{*}$ bifurcates into small amplitude periodic solution of Hopf type the value of $\tau_{1}$ is given by following equation

$$
\tau_{1 k}=\frac{1}{\eta_{0}}\left[\cos ^{-1}\left(\frac{\eta_{0}{ }^{2}\left(Q_{4} \eta_{0}{ }^{2}-Q_{2} Q_{4}\right)+\left(Q_{1} \eta_{0}{ }^{2}-Q_{6}\right)\left(Q_{5}-Q_{3} \eta_{0}{ }^{2}\right)}{\eta_{0}{ }^{2} Q_{4}{ }^{2}+\left(Q_{5}-Q_{3} \eta_{0}{ }^{2}\right)^{2}}\right)\right]+\frac{2 k \pi}{\eta_{0}}, k=0,1,2,3 \ldots
$$




\section{Numerical Simulation}

Analytic studies can never be complete without numerical verification of the results. In this section, we present computer simulation of some solutions of the system (1) and (16). Besides verification of our analytical findings, these numerical solutions are very important from practical point of view. Since the stability criteria in the absence of delay $(\tau=0)$ will not necessarily guarantee the stability in presence of delay $(\tau \neq 0)$. To illustrate the results, we choose parameters hypothetically for system (2) as

$$
c_{1}=1, \quad c_{2}=11, \quad p_{1}=10, \quad p_{2}=2, \quad q_{1}=1.5, \quad q_{2}=1.5 \text {. }
$$

When $\tau=0$ and $(x(0), y(0), z(0))=(0.63,0.2,0.2)$, with the above set of parameters values system (2) has interior equilibrium point $E^{*}\left(x^{*}, y^{*}, z^{*}\right)=(0.51622,0.398704, .0 .230213)$, which is locally asymptotically stable. All the conditions regarding to the local stability $A_{1}=3.4433>0, A_{1} A_{2}-A_{3}=16.3821>0$ are satisfied. The eigenvalues for the system are as follows -0.4646814 and $-1.48931 \pm i 1.29694$.

The results of numerical simulation are displayed graphically, in figure (1) the prey, middle predator and top predator populations are plotted against time. From figure (1) it is noted for given initial values both the populations tend to their corresponding value of equilibrium point $E^{*}$ and hence coexist in the form of steady state assuring local stability of $E^{*}$.

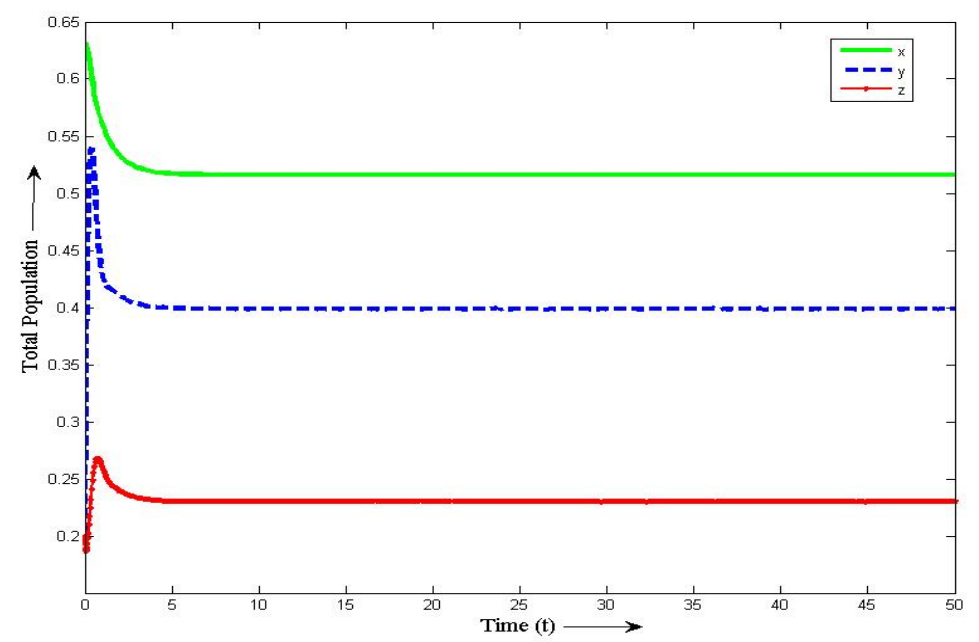

Fig. 1, Stable behaviour of $x, y$ and $z$ with $t$ when and all other parameters are same as in (25)

\section{1a Hopf Bifurcation:}

By using Liu's criterion it is interesting to observe that, when the coefficient $C_{2}$ of predator species is increased, the positive equilibrium loses its stability and a Hopf bifurcation occurs when $C_{2}$ passes a critical value. With the same choices of parameters as in (25) if $C_{2}=15.7$, then it is shown in figure 2(a), $E^{*}$ is locally asymptotically stable. Now if we increase the value of $C_{2}$, keeping other parameters fixed the stability behaviour of the system (2) changes at the bifurcation value $C_{2}^{*}=15.81$. For $c_{2}=16.7>c_{2}^{*}, E^{*}$ becomes unstable. Large amplitude of oscillations is found for this value of $c_{2}$ shown in figure 2(c-e). Figure 2(b), shows stable limit cycle for $C_{2}=15.7<C_{2}^{*}$, approaching the equilibrium point. Figure 2(f), shows periodic orbit near $E^{*}$ for $c_{2}=16.7>c_{2}^{*}$.

Thus the numerical study presented here shows that, using parameter as a control, it is possible to break unstable behaviour of the system (2) and drive it to stable state. 


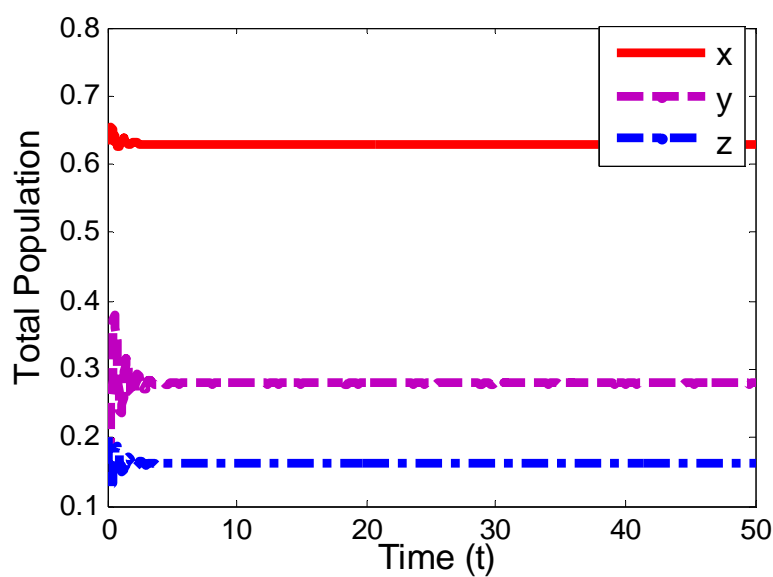

(a)

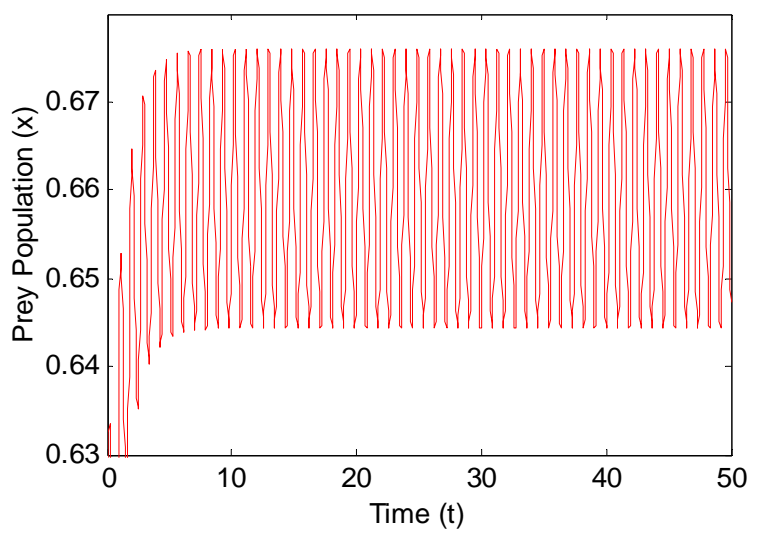

(c)

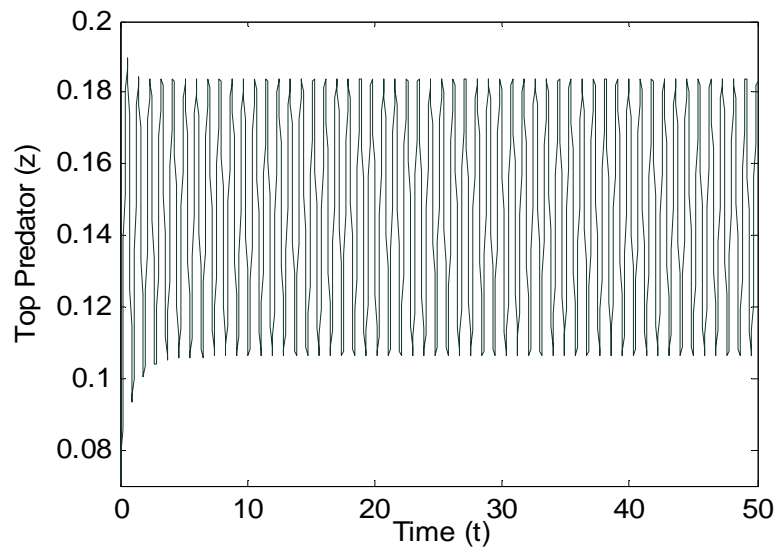

(e)

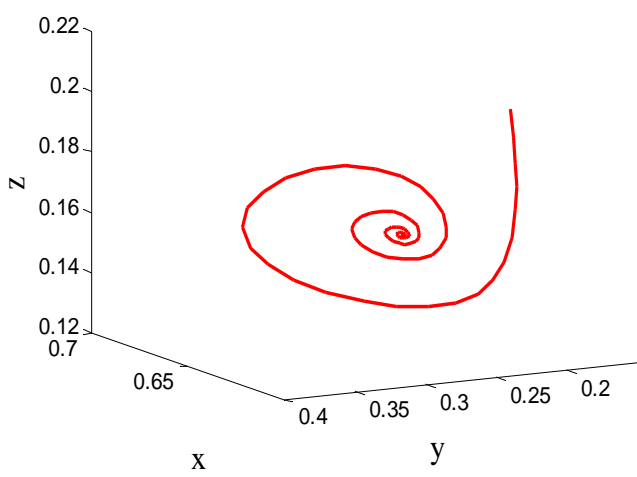

(b)

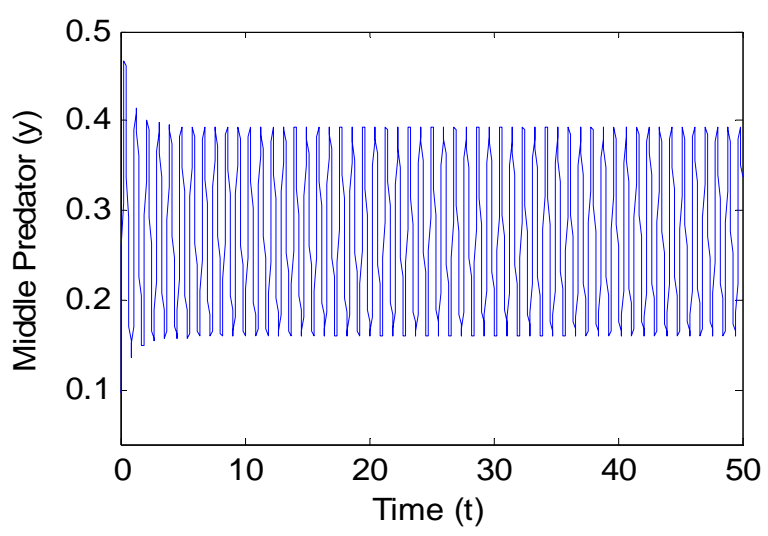

(d)

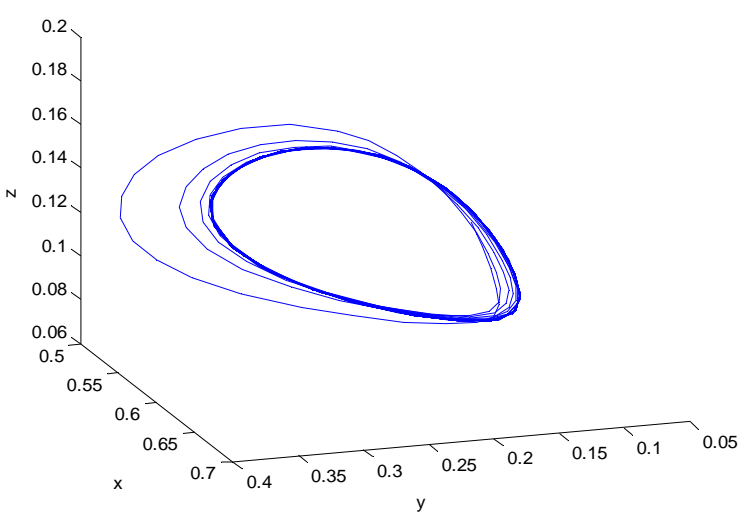

(f)

Figure 2(a), shows stable behaviour of $x, y$ and $z$ when $c_{2}=15.7$, and all other parameters are same as in (25). Fig. 2(b), gives phase portrait of system (2) showing stability. Fig 2(c-d) shows large amplitude of oscillations for $c_{2}=16.7>c_{2}^{*}$, and fig. 2(f), shows a periodic orbit near $E^{*}$.

\section{1b Type I Delay}

We see that $E^{*}$ is locally asymptotically stable in the absence delay. Now for the same set of parameters as in (25), we found that $P_{1}>0$ and $P_{3}<0$, which indicates that there exist a positive root $\omega_{0}=1.4040$. With this value of $\omega_{0}$ we calculate the critical value of delay constant $\tau=\tau_{0}=0.9820$, where the stability switch may occur. Stability switch in our case stand for switching from stable steady state to stable oscillatory state. We also observe that the transversal condition (20) is satisfied as 
$\operatorname{Re}\left(\frac{d \lambda}{d \tau}\right)^{-1}=14.3744>0$

Therefore $E^{*}\left(x^{*}, y^{*}, z^{*}\right)$ loses its stability as $\tau$ passes through critical value $\tau_{0}$. It is shown in figure $3\left(\right.$ a) that if $\tau=0.8<\tau_{0}$, $E^{*}$ is locally asymptotically stable. But if we take $\tau=1.1>\tau_{0}, E^{*}$ is found to be unstable and bifurcating periodic oscillation near $E^{*}$ is observed .Thus, we conclude that time delay breaks the stable behaviour of system (2) and drives it to an unstable state. We have drawn time series in figure 3(b) for $\tau=\tau_{0}=0.9820$ and figure 3(c-e) for $\tau=1.1>\tau_{0}$. Large amplitude oscillations are observed for this value of $\tau$.

We have studied phase portrait of the delayed system for various value of delay. Figure 3(f) shows phase portrait of system for $\tau=0.8<\tau_{0}$. It shows stable limit cycle approaching the equilibrium point. Figure 3(g) depicts the stable limit cycle due to Hopf bifurcation at $\tau=\tau_{0}=0.9820$. Figure 3(h) shows phase portrait of system for $\tau=1.1>\tau_{0}$. It demonstrates a periodic orbit near $E^{*}$.Further on increasing the value of delay we have observed the strange attractor for $\tau=2.2$, which displayed in figure 3(i).
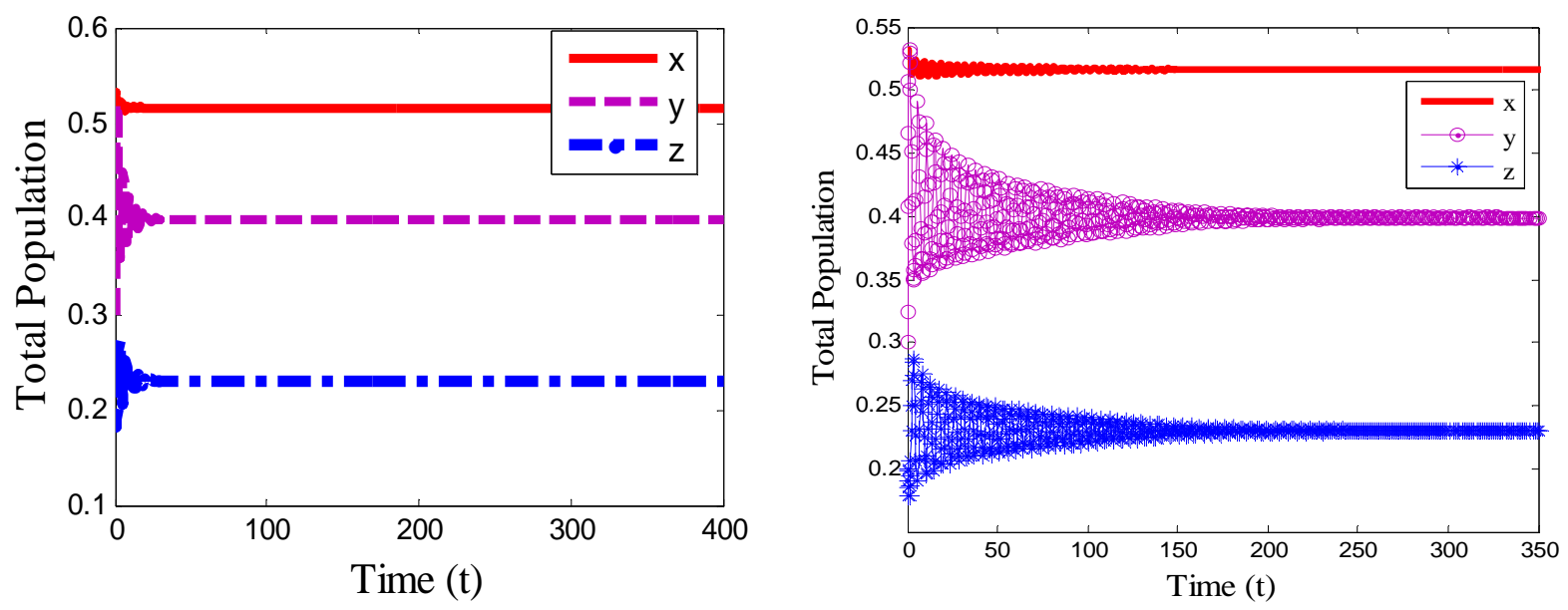

(a) (b)

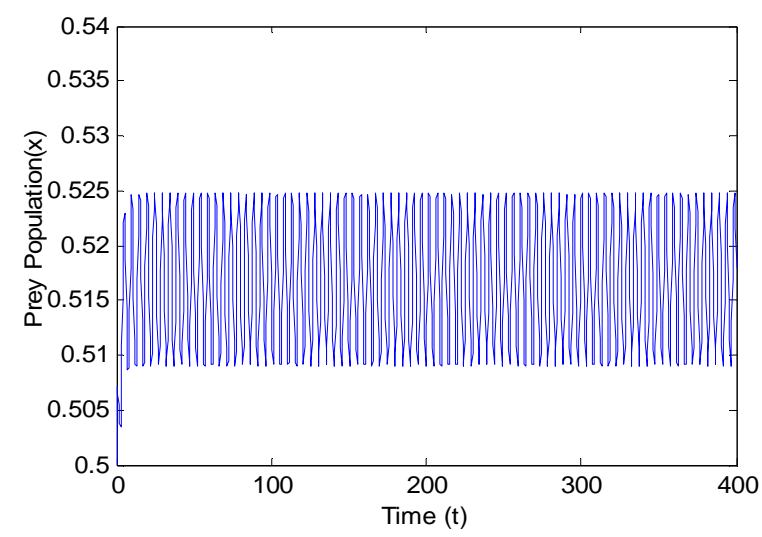

(c)

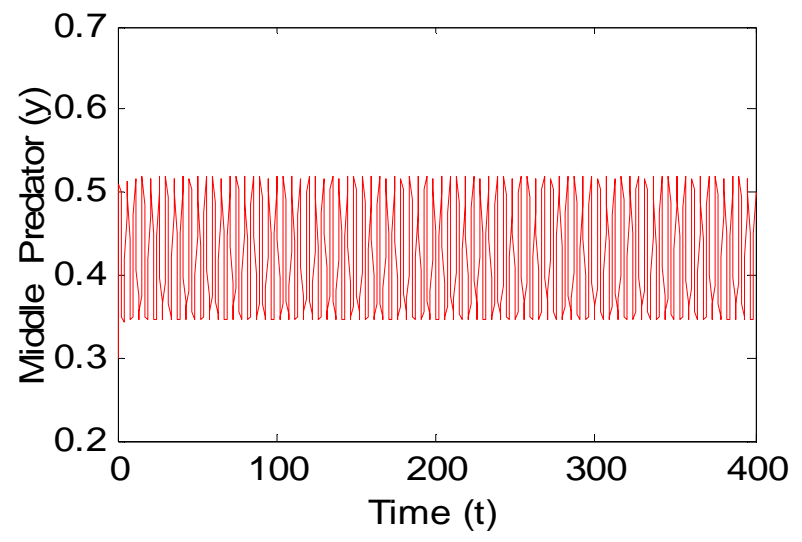

(d) 


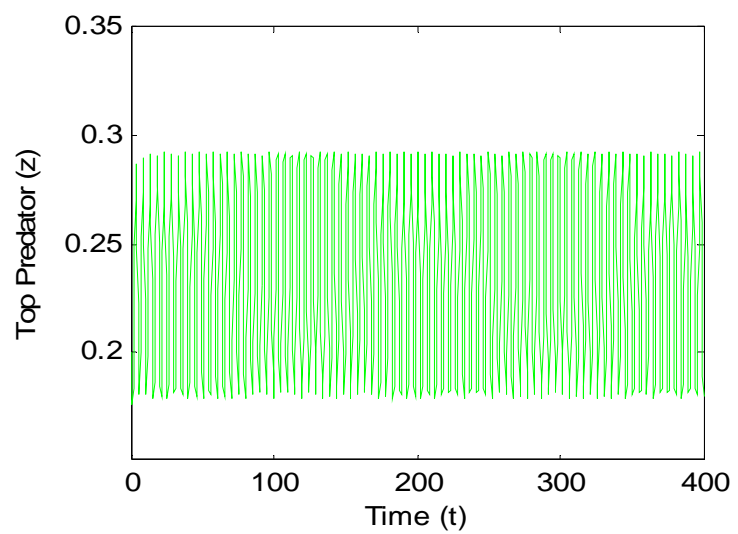

(e)

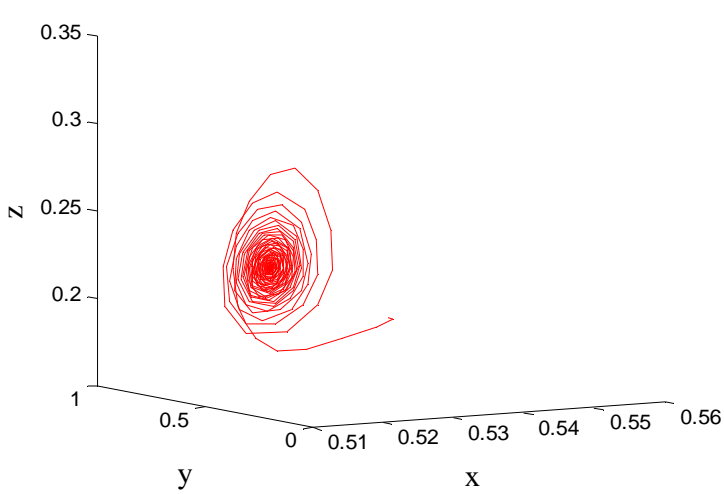

(g)

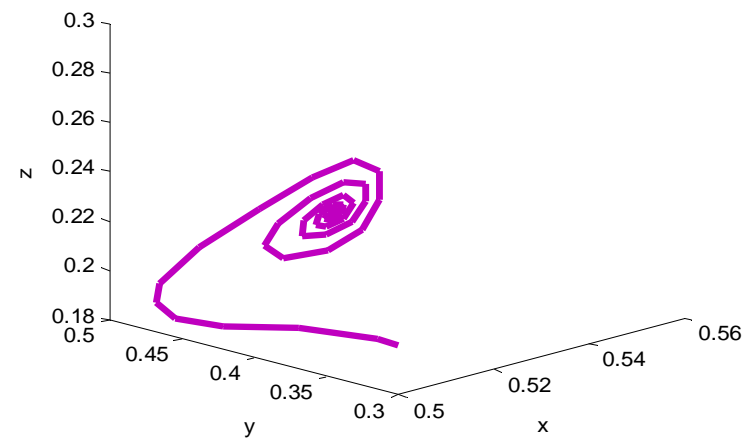

(f)

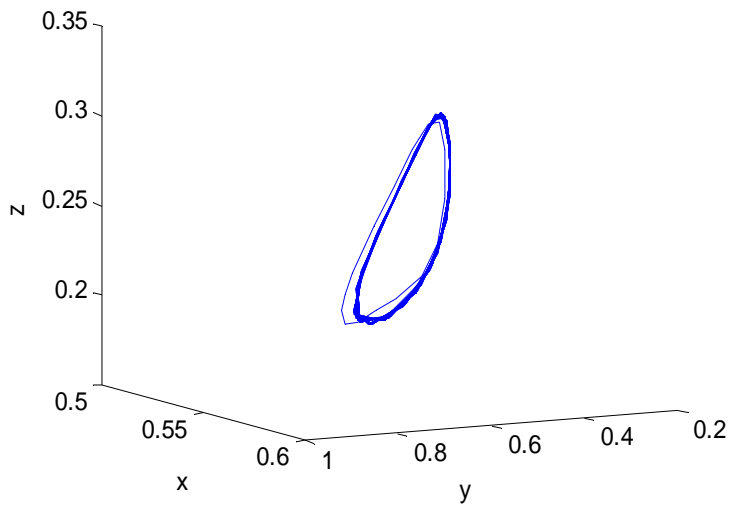

(h)

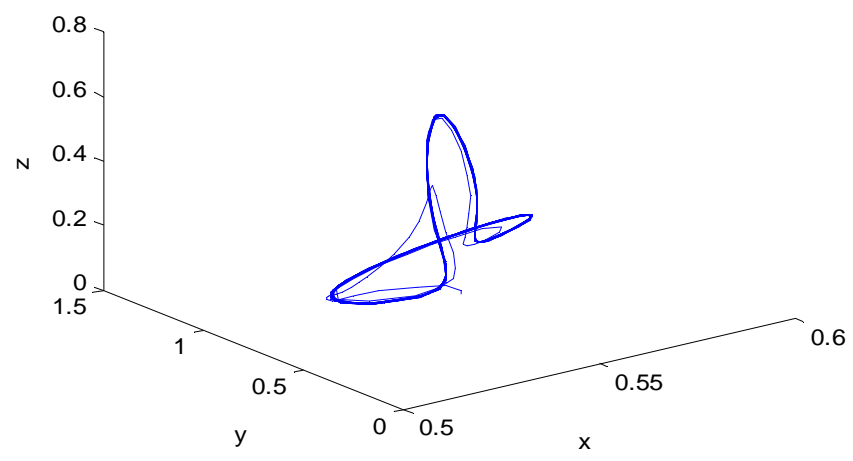

(i)

Fig. 3(a), Shows stable behavior of $x, y$ and $z$ in finite time and fig. 3(f), shows stable limit cycle when $\tau=0.8<\tau_{0}$ and all other parameter are same as (25). Small amplitude of oscillations is observed in fig. 3(b) and Fig. 3(g) shows limit cycle at $\tau_{0}=0.9820$. Fig. 3(c)-(e) shows large amplitude of oscillations for $\tau=1.1>\tau_{0}$ and Fig. 3(h), gives periodic orbit near $E^{*}$. Fig. 3(i), shows strange attractor at $\tau=2.2$.

\section{1c Type II Delay}

We observed that there is no effect of delay in the system (21) for above set of parameters given in (25). Therefore in order to study the delayed system we consider another set of parameters given as

$c_{1}=2, \quad p_{1}=3.1, \quad c_{2}=5.3, \quad q_{1}=0.3, \quad p_{2}=2, \quad q_{2}=0.6$.

With the same choices of parameters as in (26), we observed that there exist a unique interior equilibrium point given by $x^{*}=0.3506546, y^{*}=0.1293336$ and $z^{*}=0.1975647$. Simulation of the model with this set of parameter without delay 
gives stable dynamics as presented in figure 4(a).With the same set of parameters, we found that $S_{1}>0$ and $S_{3}<0$ which indicates that there exist unique positive root, given by $\omega_{0}=0.1582$ and therefore critical value of delay $\tau=\tau_{1}=6.7544$ for $k=0$. Therefore $E^{*}\left(x^{*}, y^{*}, z^{*}\right)$ loses its stability as $\tau$ passes through critical value $\tau_{1}$. It is shown in figure 4(b) that if $\tau=6.6<\tau_{1}, E^{*}$ is locally asymptotically stable. But if we take $\tau=7.0>\tau_{1}, E^{*}$ is found to be unstable and bifurcating periodic oscillation near $E^{*}$ is observed shown in Fig. 4(d-f). Thus we conclude that time delay breaks the stable behaviour of system (2) and drives it to an unstable state. Small amplitude of oscillations are observed at $\tau=\tau_{1}=6.7544$, shown in figure 4(c). In figure 4 (f), we see phase portrait for $\tau=6.6<\tau_{1}$ it shown a limit cycle. But for $\tau=7.0>\tau_{1}$, figure 4 (g) shows a periodic orbit near $E^{*}$. We also obtained a strange attractor on increasing the value of delay up to $\tau=15$, Shown in figure 4(i).

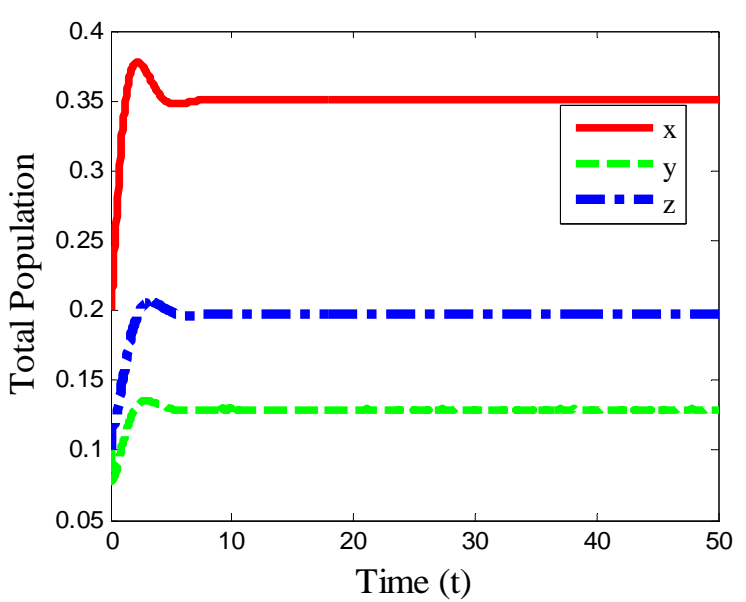

(a)

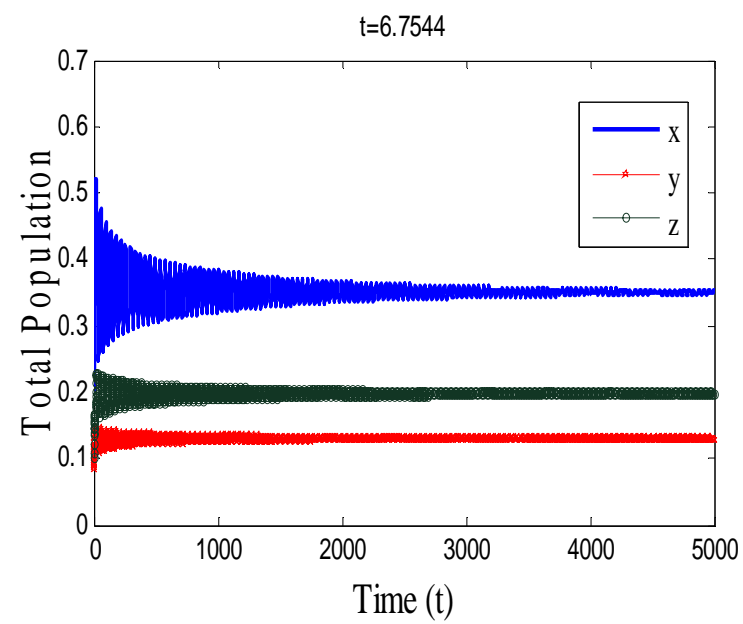

(c)

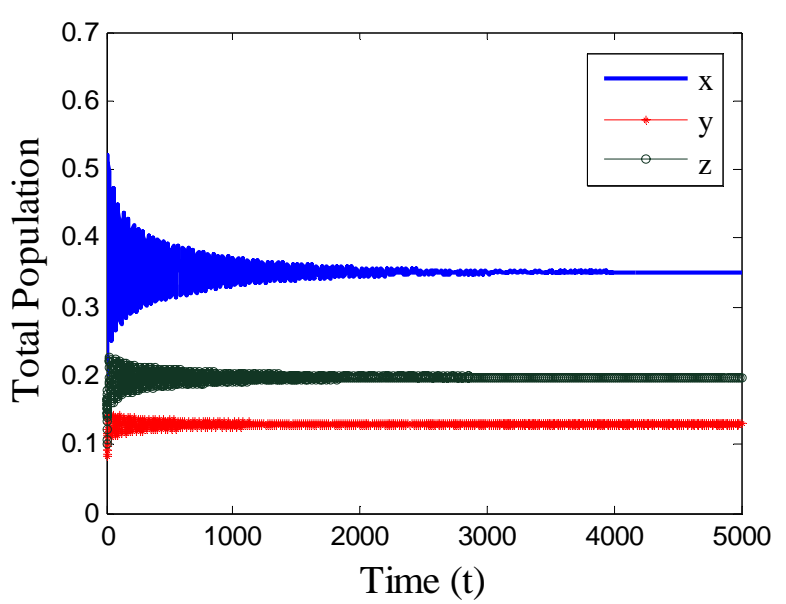

(b)

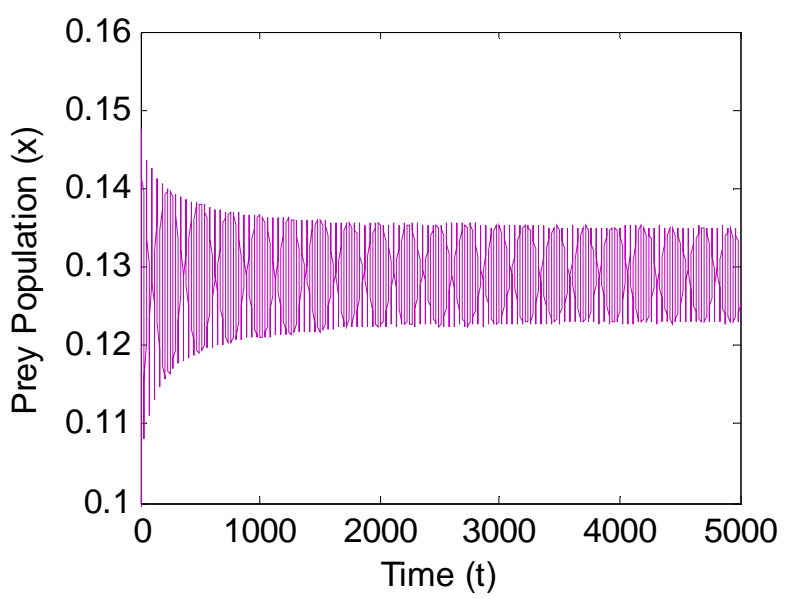

(d) 


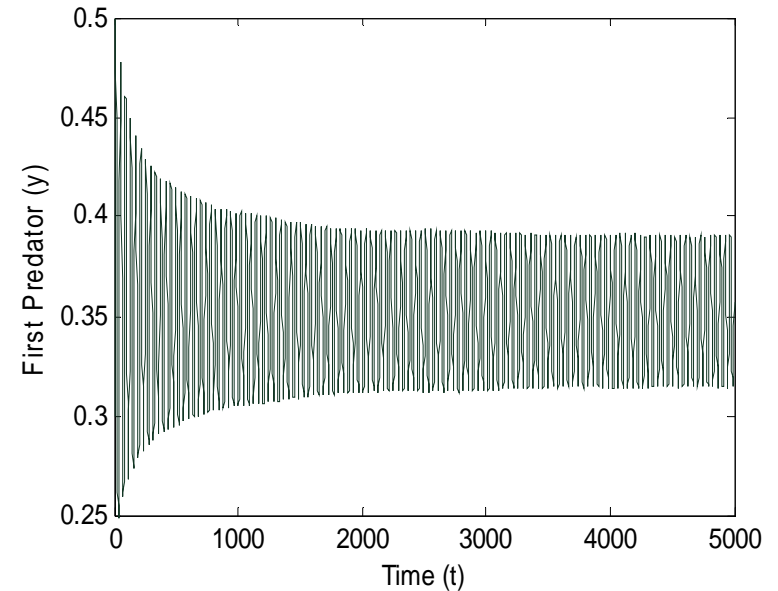

(e)

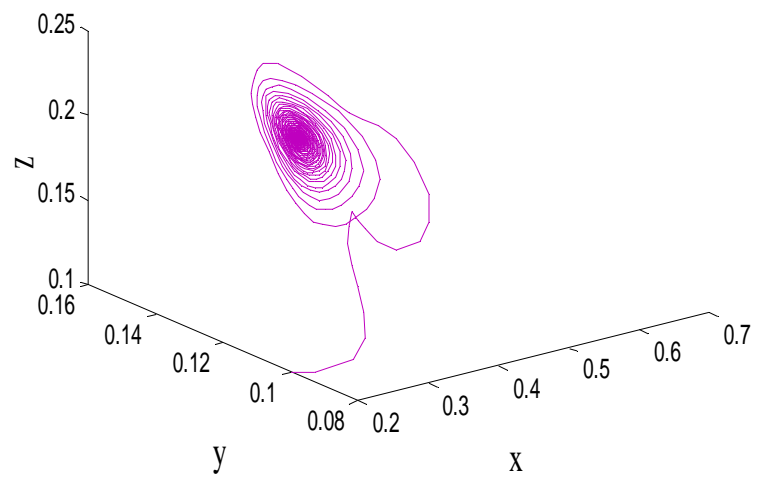

(g)

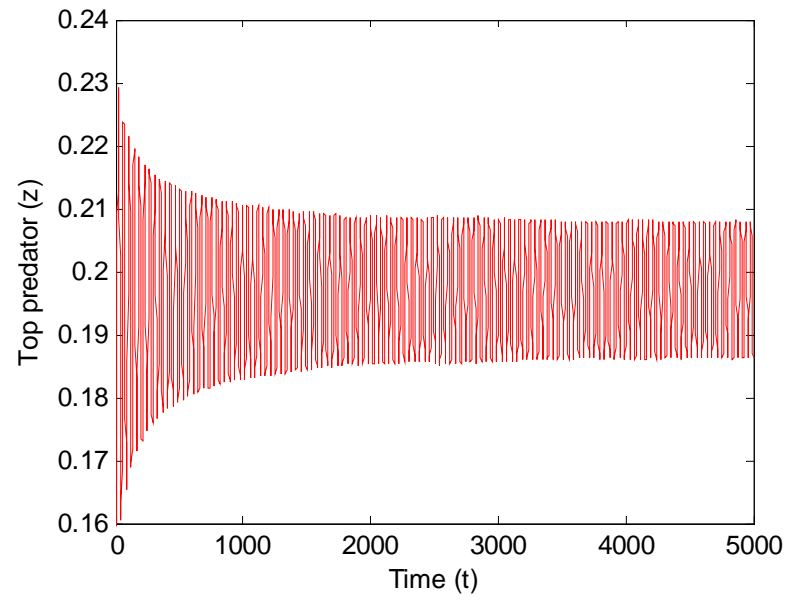

(f)

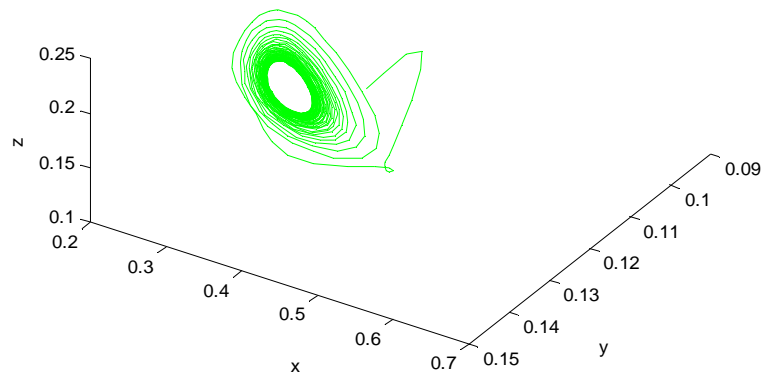

(h)

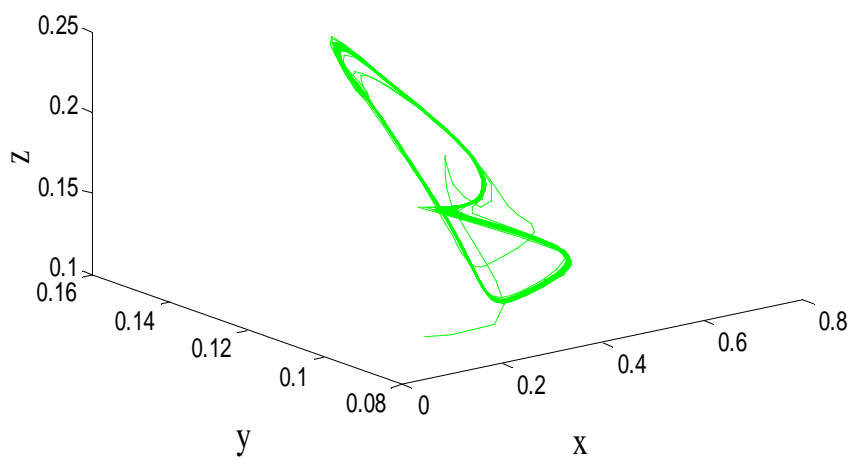

(i)

Fig. 4(a), Shows stable behavior of $x, y$ and $z$ when $\tau=0$. Fig.4(b), 4(g) Shows stability of $E^{*}$ and phase portrait of the system (21) showing stability when $\tau=6.6<\tau_{1}$.. Small amplitude of oscillations is observed at $\tau=\tau_{1}=6.7544$ shown in fig.4(c). Fig. 4(d)-(f) shows unstable behavior for $\tau=7.0>\tau_{1}$. Fig. 4(g), gives periodic orbit near $E^{*}$ for $\tau=7.0>\tau_{1}$ and all other parameter values are same as (26). Fig. (i), shows strange attractor at $\tau=15$. 
With the same set of parameters values as (25), we found that when top predators are absent, prey and middle predators exists. In figure (5), we obtained that in the presence of top predator population, prey population increases and population of middle predator decreases, but in the absence of top predator population of prey decreases and middle predator population increases.

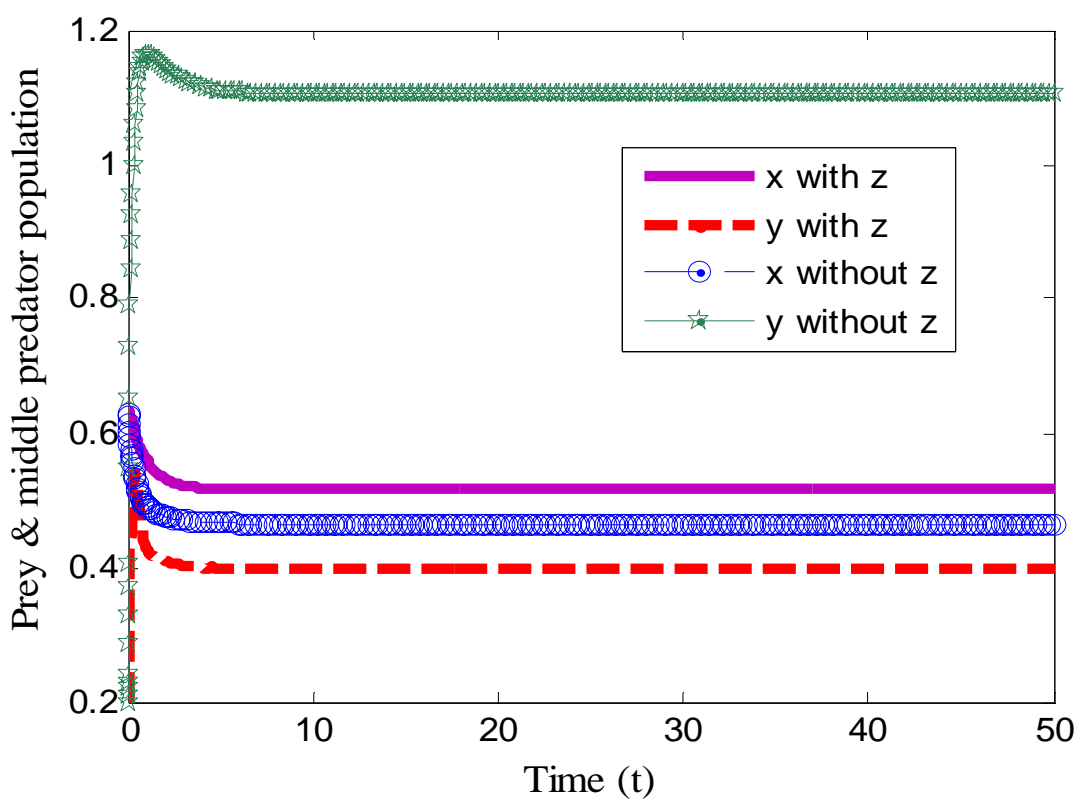

Fig. 5, comparison of prey and middle predator populations with and without top predator with the same set of parameters values as (25)

\section{Conclusion}

In this paper, we studied the dynamical behaviour of a tritrophic food chain model. Ratio-dependent type III functional response is considered to model the interactions among the species of the system. It is shown (in theorem 3.1) that non-dimensionalized system (2) is uniformly bounded, which, in turn, implies that the system is biologically well behaved. It has been recognized that most of the studies of continuous time deterministic models revel two basic patterns: approach to equilibrium or to a limit cycle. The basic rationale behind such type of analysis was the implicit assumption that most food chains we observe in nature correspond to stable equilibrium point $E^{*}\left(x^{*}, y^{*}, z^{*}\right)$. From this view point, we have presented the stability and bifurcation analysis of most important equilibrium point $E^{*}\left(x^{*}, y^{*}, z^{*}\right)$. From numerical calculations, in figure (5), we observed that when top predators are absent, predator $y$ and prey $x$ co-exist. From this we conclude that in presence of top predators, prey population increases and causes depression in the middle predator but in the absence of top predator prey population goes on decreasing and middle predator increases. Thus our model may be looked upon as the mathematical model for tea plant - pest (e.g. Looper Caterpillar) - beneficial predator (natural enemy of the pest e.g. Sarcophaga). These types of models may be used for the purpose of 'Bio-Control of Pests' which is suggested by several researchers to reduce the hazards of chemical pesticides (Das et al. 1988) and (Kabir 2001).

Time-delay has a significant impact on stability. It is necessary to take into account the effect of time-delay to have a biologically useful mathematical model [MacDonald, 1989; Gopalsamy, 1992; Kuang, 1993]. From this view point, we have formulated the model (16) where the delay may be looked upon as the gestation period or reaction time of the top predator. But in model (21), we have assumed that the change rate of the top predator depends on the number of middle predators and top-predators present at some previous time. Then the rigorous analysis leads us to the fact that the stability criterion in absence of delay is no longer enough to guarantee the stability in presence of delay. It is found that when time delay is absent, system is uniformly bounded, which in turn implies that the system is biologically well behaved. We have found a critical value of time delay $\tau_{0}$ for the model (16) and $\tau_{1}$ for the model (21) such that if time delay is less than $\tau_{0}$ system (16) is stable and if time delay exceeds $\tau_{0}$ then system becomes unstable and show periodic oscillations. System (21) is stable for $\tau<\tau_{1}$ and unstable for $\tau>\tau_{1}$. This implies that time delay plays a significant role on the stability of the system. It breaks the stable behavior of system and drives it to unstable state. All our important mathematical findings and graphical representation of variety of solutions of system (2), (16) and (21) are depicted by using MATLAB programming. 


\section{References}

Abrams P. A. and Ginzburg L. R., 2000. The nature of predation: prey dependent, ratio-dependent or neither. Trends in Ecology and Evolution, Vol. 15, pp. 337-341.

Arditi R. and Ginzburg L. R., 1989. Coupling in predator-prey dynamics: ratio-dependence. J. Theor. Biol., Vol. 139, pp.311-326.

Berezovskaya F., Karev G. and Arditi R., 2001. Parametric analysis of the ratio-dependent predator- prey model. J. Math.Biol., Vol. 43, pp. 221-246.

Freedman H. I. and Waltman P., 1977. Mathematical analysis of some three-species food-chain models, Math. Biosci., Vol. 33, pp. 257-276.

Fritzsche-Hoballah M., Turlings T., 2001. Experimental evidence that plants under caterpillar attack may benefit from attracting parasitoids, Evol. Ecol. Res., Vol. 3, pp.1-13.

Gopalsamy K., 1992. Stability and Oscillations in Delay Differential Equations of Population Dynamics, Kluwer Academics, Dordrecht.

Gomez J. and Zamora R., 1994. Top-down effects in a tritrophic system: parasitoids enhance plant fitness, Ecology, Vol.75, pp.1023-1030.

Hsu S. B., Hwang T. W. and Kuang Y., 2003. A ratio-dependent food chain model and its applications to biological control. Mathematical Biosciences, Vol.181, pp. 55-83.

Jost C. and Ellner S. P., 2000. Testing for predator dependence in predator-prey dynamics: a non- parameteric approach. Proc. $R$. Soc. Lond. B, Vol. 267, pp. 1611-1620.

Kara R. and Can M., 2006. Ratio-dependent food chain models with three trophic levels. Int.J. of Comp.Sci., Vol. 1/2, pp. 85-92.

Kuang Y., 1993. Delay Differential Equations with Applications in Population Dynamics, Academic Press, New York.

Kuang Y. and Beretta E., 1998. Global qualitative analysis of a ratio-dependent predator-prey system, J. Math. Biol.,Vol. 36, pp. 389-406.

Li B. and Kuang Y., 2000. Simple food chain in a chemostat with distinct removal rates, J. Math. Anal. Appl., Vol. 242, pp.7592.

Lotka A., 1925. Elements of Physical Biology. Williams and Wilkins, Baltimore.

Loon J. V., Boer G.De. and Dicke M., 2000. Parasitoid-plant mutualism: parasitoid attack of herbivore increases plant reproduction, Entomol. Exp. Appl., Vol. 97, pp. 219-227.

Moghadas S. M. and Gummel A. B., 2003. Dynamical and numerical analyses of a generalized food chain model. Applied math. And comp., Vol. 142(I), pp. 35-49.

Maiti A. and G. P. Samanta G. P., 2005. Complex dynamics of a food chain model with mixed selection of functional responses, Bull. Cal. Math. Soc., Vol. 97, pp. 393-41.

Wangersky P. J. and Cunnigham W. J., 1957. Time lag in prey-predator population models. Ecology, Vol. 38, pp. 136-139.

Das S.C., Borthakur M. C., and Gope B., 1988. Need for non-conventional approach in tea pest management in: Proceedings of the 30th Tocklai Conference, pp. 80-89.

Kabir S. E., 2001. Non-conventional methods of pest control in tea (Camellia sinensis L.), in: S.K. Ghoshal S. K., Ray D. (Eds.), Recent Advances in Animal Science Research, Orion Press International, West Bengal, Vol. 1 pp. 163-167.

\section{Biographical notes}

Prof. Manju Agarwal has been working in the field of Mathematical Biology for the last 30 years. Her major research interests are in Fluid dynamics and Mathematical modeling in ecology, environment and epidemiology. She has 94 research publications in standard scientific journals of International and National level. She has supervised more than a dozen Ph.D. theses of University of Lucknow. At present, she is Head of the Department of Mathematics and Astronomy, Lucknow University, Lucknow. She is member of Internal Quality Assurance Cell of the University and life member of various mathematical societies like National Academy of Sciences, International Academy of Physical Sciences, The Indian Society of Industrial and applicable Mathematics and the International Federation of Nonlinear Analysts (IFNA).

Vimlesh Singh is working as a student under the supervision of Prof. Manju Agarwal.

Received September 2012

Accepted December 2012

Final acceptance in revised form May 2013 